\title{
Laser Liposuction-Skin Tightening in Body Contouring of Massive Weight Loss Patients
}

\author{
MOUSTAFA S.A. MEKY, M.D. \\ The Department of Plastic Surgery, Faculty of Medicine, Al-Azhar University, Cairo, Egypt
}

\begin{abstract}
Background: Significant weight loss patients have diverse soft tissue laxity that involve every area of their body. The aim of surgical interventions is to help these patients regain their form, function, and most importantly through minimal scars. The traditional excisional procedures inevitably end up with multiple noticeable scars that have mostly equal concern and complain to pre-excision soft tissue laxity. LAL is a bet new modality that proved its efficacy in reducing fat and induce skin tightening Non-MWL patients. The study her proved its efficacy in MWL patients.
\end{abstract}

Material and Methods: The study was classified into 2 time-period related groups. They were before and after introduction of LAL in the author's practice. The study was conducted over 537 post MWL patients (194 and 343 patients in $1^{\text {st }}$ and $2^{\text {nd }}$ groups respectively). The number of total procedures for all were 1235 procedures. The follow-up period was from $5 \mathrm{M}-2 \mathrm{Y}$. The number of bariatric to non-bariatric weight loss in the first group was 89:105 (45.9\%: $54.1 \%)$. In the second group, it was 102:241 (bariatric: non-bariatric weight loss) in a percentage of $(29.7 \%: 70.3 \%)$.

Results: There was no significant difference in the age, weight, and regional skin redundancy and subcutaneous fat thickness in the baseline between two groups ( $p 0.05)$. When analyzing the data it was found that, 1- The number of MWL patients presented for body contouring increased after the author introduced LAL in his work, 2- A reversal in number of patients that had loss of weight through bariatric surgery and number of patients who lost weight through diet and exercise between $1^{\text {st }}$ and $2^{\text {nd }}$ groups (112/82:136/207), 3The second group patients tended to have multiple procedures comparing to first group patients n - 276 (1.4) Vs n - 959 (2.9), 4- There was a little variable difference in number of procedures between patients that have loss of weight through bariatric and diet and exercise in either group (1.6-2.3 versus 2.8-4.3), 5- The number of excisional procedures was decreased in the second group (33\% vs $77.5 \%)$, 6- The overall patients' satisfaction showed variable results with $(81 \%)$ of second group being very satisfied in contrast to $(55.5 \%)$ in first group, 7- The number of non-bariatric weight loss was higher in the second group (73.8\% Vs 55.6\%), 8- Improvement in skin appearance was more higher in the $1^{\text {st }}$ than $2^{\text {nd }}$ group $(79.3 \%$ Vs $72.2 \%)$.

Conclusion: The outcome of this study proved the efficacy and safety of LAL-ST in post MWL patients.
Key Words: Laser liposuction - Skin tightening - Body Contouring.

\section{INTRODUCTION}

Massive weight loss (MWL) patients are special aesthetic group that need prudent decision for surgical intervention. They have variable degrees of skin redundancy and residual fat that in common leave external weaving and disfigurement. The will known procedures for removing redundant skin-excess fat complex is surgical excision [17]. These excisional procedures always leave much scars affecting different areas of the body, replacing the stigmata of skin redundancy by stigmata of scars. This render some patient to avoid exposure to such excisional procedures, some others may have limited excisional procedures.

Laser liposuction and skin tightening (LALST) is now a commonly used modality for removal of unwanted fat and moreover skin reduction. Since its FDA approved in 2006, studies $[\mathbf{7 , 8 , 1 1}$ ] have continued to support early clinical observations of decreased adiposity, shorter recovery times, and improved skin shrinkage. The laser lipolysis mechanism of action is liquefaction of fat, coagulation of small blood vessels, increased fibroblast numbers, and stimulation of new collagen formation with subsequent skin tightening and augmentation of tissue elasticity.

Radiofrequency and cryolipolysis are additional modalities supporting lipolysis and collagenesis externally $[\mathbf{1 3 , 1 4 , 1 7 ]}$. However, these devices are still short of efficacy and internal application of laser energy still the most effective method of reducing subcutaneous fat and enhancing skin tightening. In this work a retrospective analysis of the author's treated post MWL patients. The aim of this retrospective analysis is to check how much the introduction of LAL-ST technique in his work 
affects the incidence of invasive excisional procedures in such group of patients.

\section{PATIENTS AND METHODS}

The study was classified into 2 groups. They were before and after LAL was introduced in the author's practice. Data analysis of 1235 procedures over 537 post MWL patients representing the 2 groups (194 and 343 patients in $1^{\text {st }}$ and $2^{\text {nd }}$ groups respectively) was undertaken. The follow-up period ranged from 5 months- 2 years. In the first group, 89 patients had MWL after bariatric surgery (45.9\%) and the rest 105 lost weight exclusively through diet and exercise (54.1\%). In the second group, 102 patients lost weight through bariatric intervention $(29.7 \%)$ and the rest 241 lost weight through diet and exercise $(70.3 \%)$.

\section{RESULTS}

There was no significant difference in the age, weight, and regional skin redundancy and subcutaneous fat thickness in the baseline between two groups $(p<0.05)$.
Data analysis revealed that, the number of MWL patients presented for body contouring increased after the author introduced LAL in his work, A reversal in number of patients that had loss of weight through bariatric surgery and number of patients who lost weight through diet and exercise between $1^{\text {st }}$ and $2^{\text {nd }}$ groups (112/82:136/207), It was noticed that the second group of patients desired to do multiple procedures than the first group patients n: 276 (1.4) versus n: 959 (2.9). There was unnoticed difference in number of procedures between patients that have post bariatric MWL and patients that have MWL through diet and exercise in either group (1.6: 2.3 versus 2.8: $4.3)$. The number of excisional procedures were decreased in the second group (33\% vs $77.5 \%$ ). The overall patients' satisfaction showed variable results with $(81 \%)$ of second group being very satisfied in contrast to $(55.5 \%)$ in first group. The number of non-bariatric MWL patients in the second group was higher in comparison to the first group $(73.8 \%$ Vs $55.6 \%)$. Improvement in skin appearance was more higher in the $1^{\text {st }}$ than $2^{\text {nd }}$ group $(79.3 \% \mathrm{Vs} 72.2 \%)$.

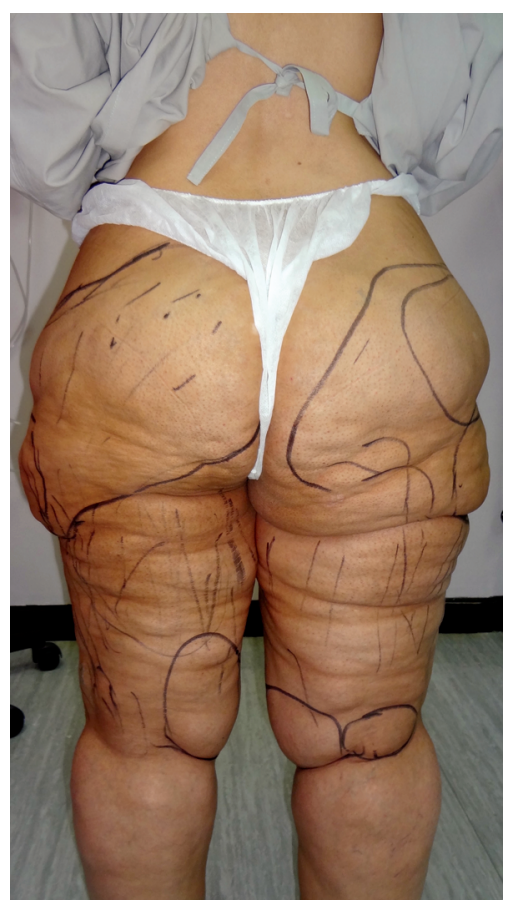

(A)

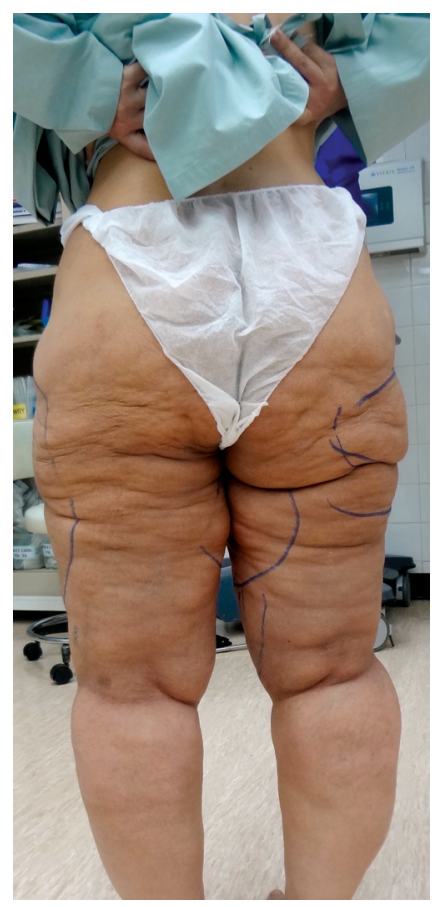

(B)

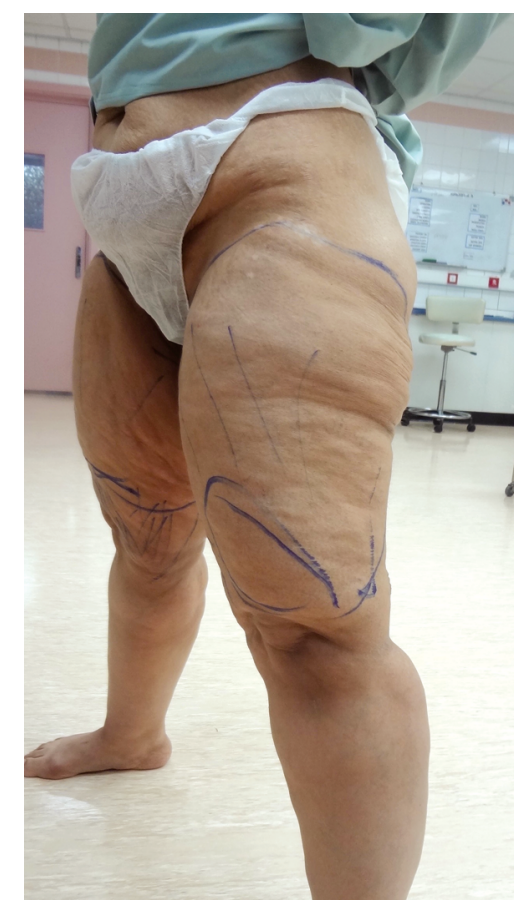

(C)

Case (1) (Group 2): Female patient 35 years old with post bariatric MWL. She has massive fat involution with moderate soft tissue sagging and external surface irregularities with disproportionate weight loss between her upper and lower body. She was exposed to 2 sessions of LAL-ST for contouring of her thighs, buttocks, and saddle bag areas. Fig. (A) shows the patient before starting the first session with marking of the target areas. Figs. (B,C) shows the same patient before starting the second session of LAL with much skin reduction and fat involution. 


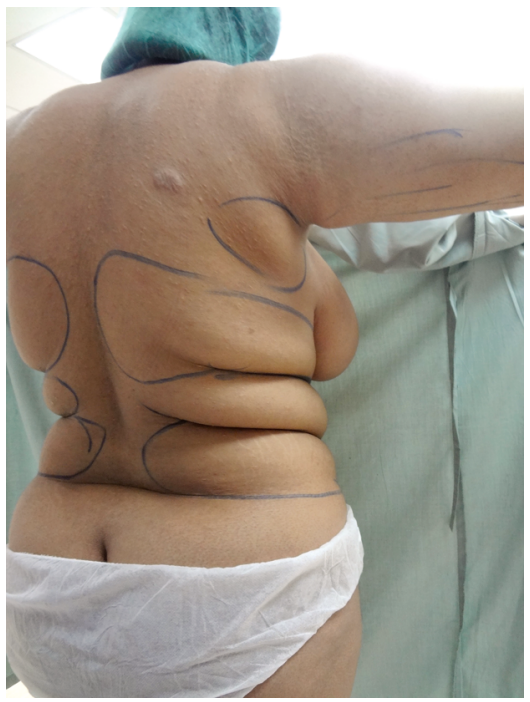

(A)

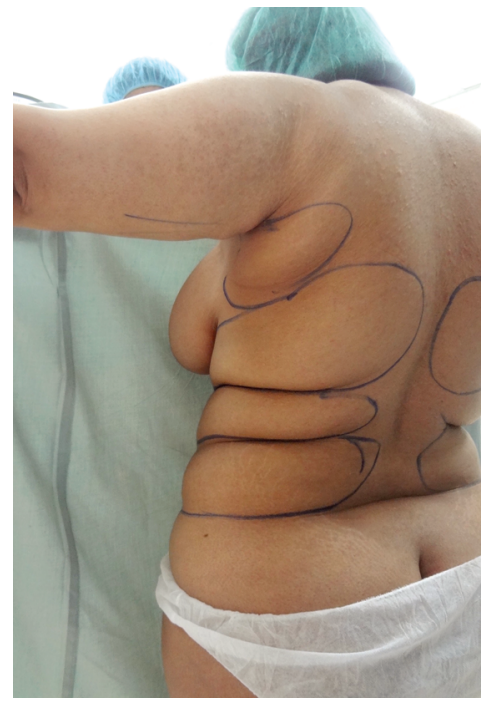

(B)

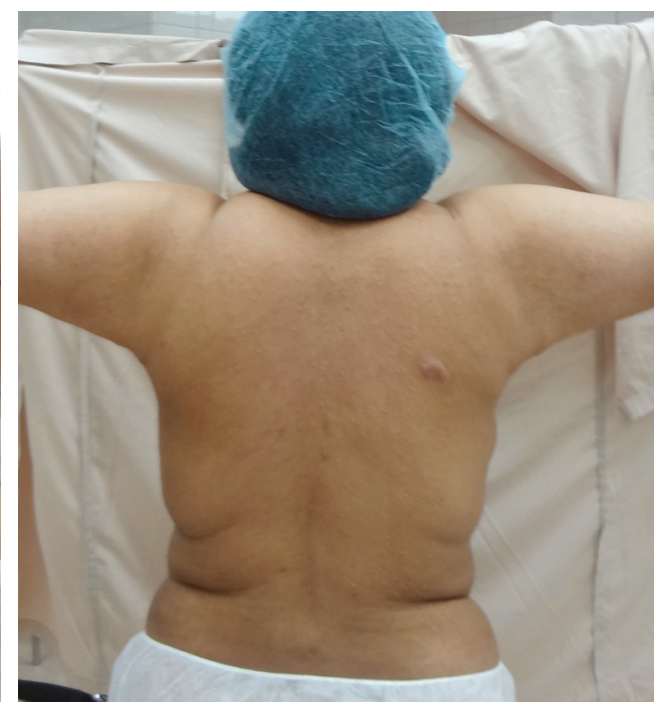

(C)

Case (2) (Group 2): Female patient 29 years old with post MWL through exercise and diet control. Figs. (A,B) shows massive axillary soft tissue laxity and back rolls redundancy. Single session of LAL-ST was done. Fig. (C) shows marked reduction of redundant skin and subcutaneous soft tissue involution.

(A)

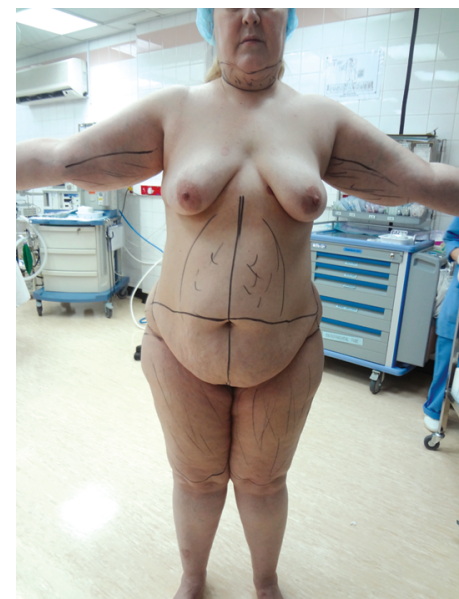

(C)

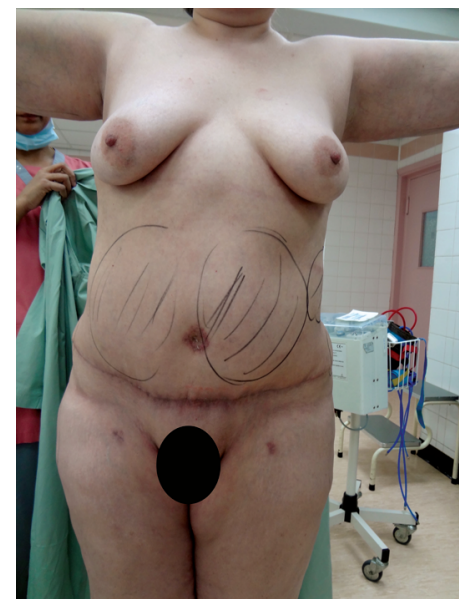

(B)

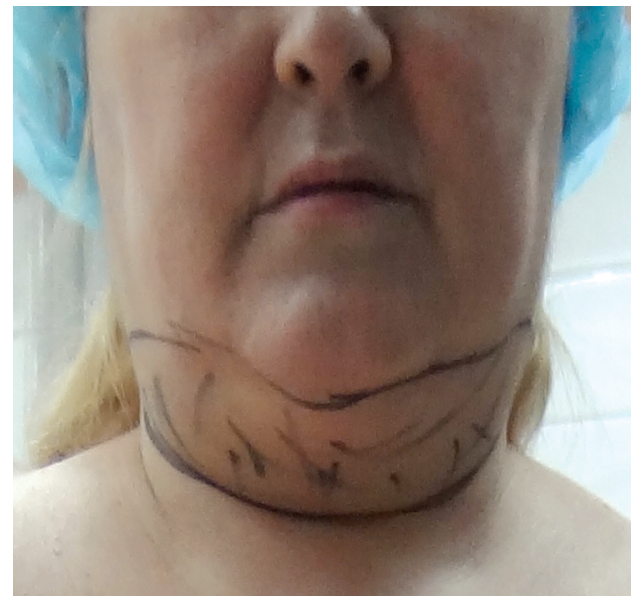

(D)

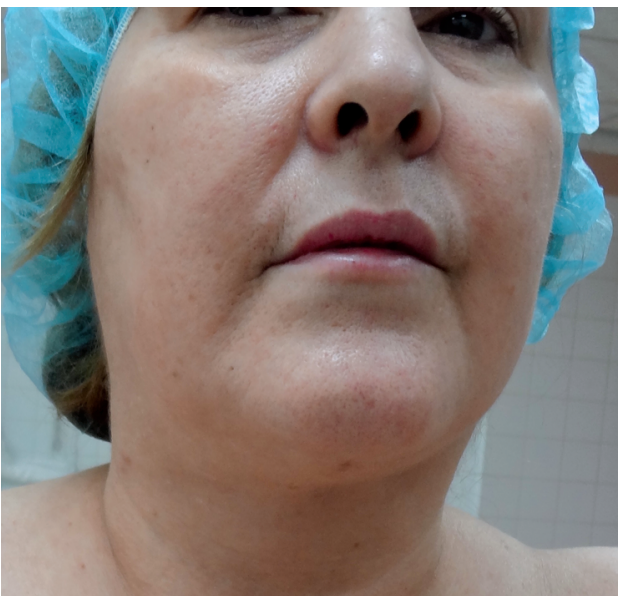

Case (3) (Group 2): Female patient 43 years old with Non-bariatric MWL. Figs. (A,B) showed that the patient had pendulous abdomen, bilateral arm ptosis, antero-medial thigh lipodystrophy, skin laxity, and cellulite, and lastly submental soft tissue laxity. Her abdominal problem was addressed through excisional surgery. The wrist of soft tissue laxity in arms, submental, and thigh regions was managed by LAL. Figs. (C,D) show the result 3 months after surgery with much improvement of the operated areas. She got much reduction of soft tissue laxity in arms, submental, and thigh regions. She had minor residual upper abdominal subcutaneous soft tissue excess that was managed by radiofrequency. 
(A)
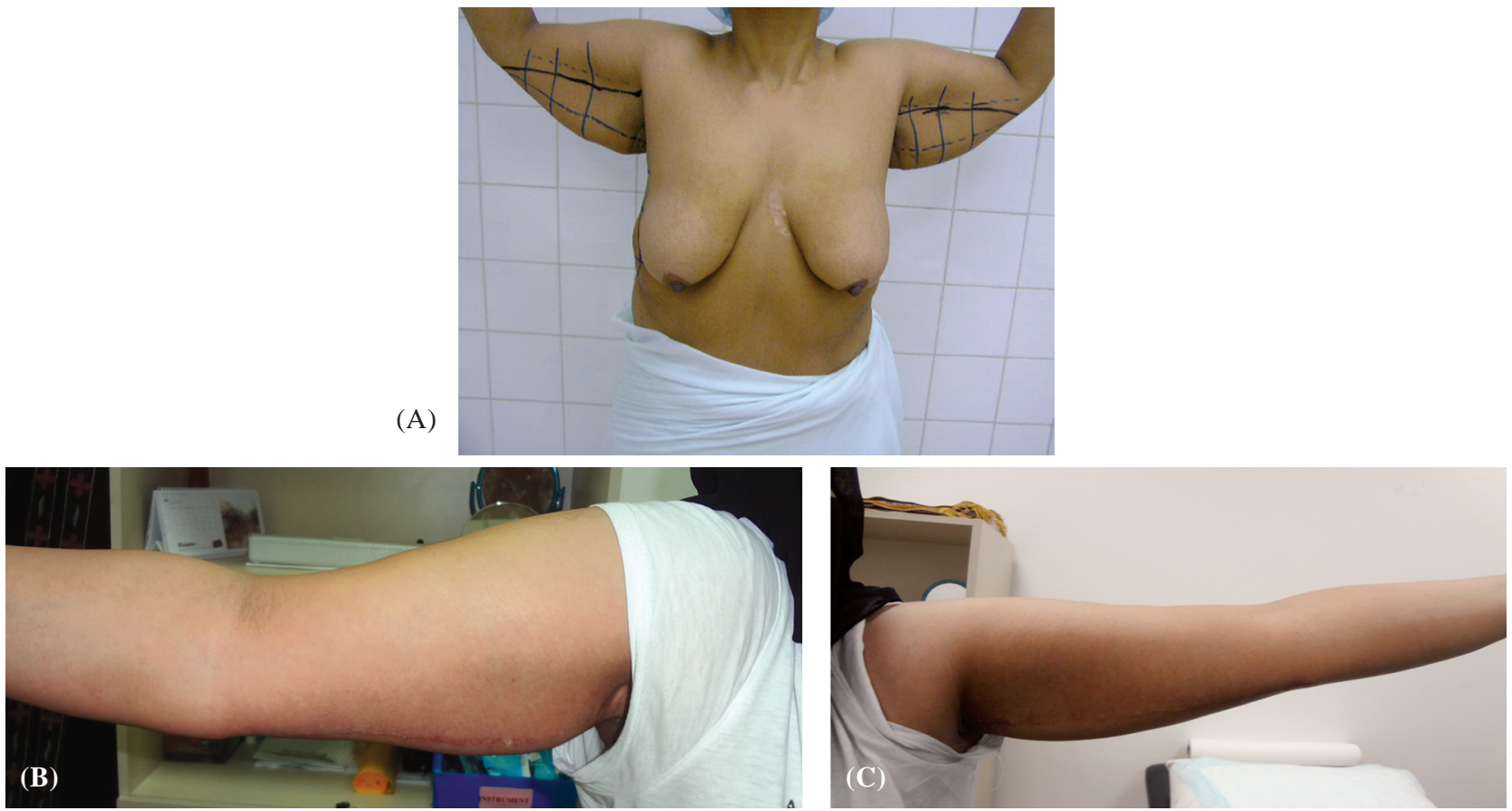

Case (4) (Group 1): Female patient 32 years old with post bariatric MWL. Fig. (A) shows bilateral high grade arm ptosis and breast ptosis. The patient preferred to address her arm ptosis in this stage. Bilateral brachioplasty through excisional surgery was done. Figs. $(B, C)$ shows post operative results 3 months after surgery.

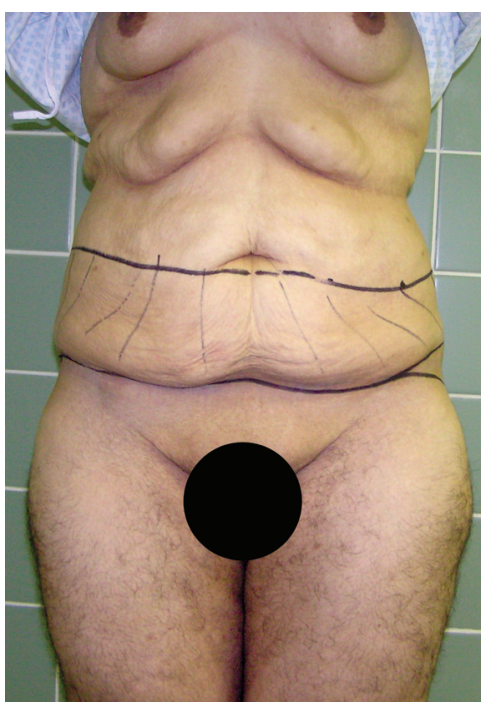

(A)

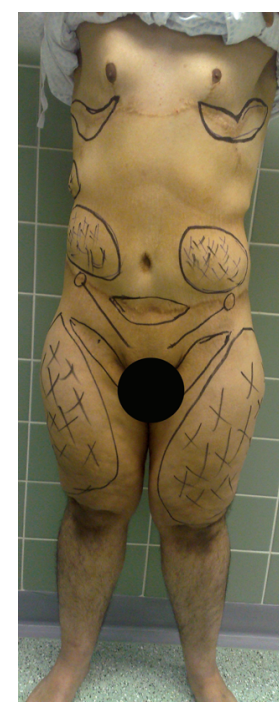

(B)

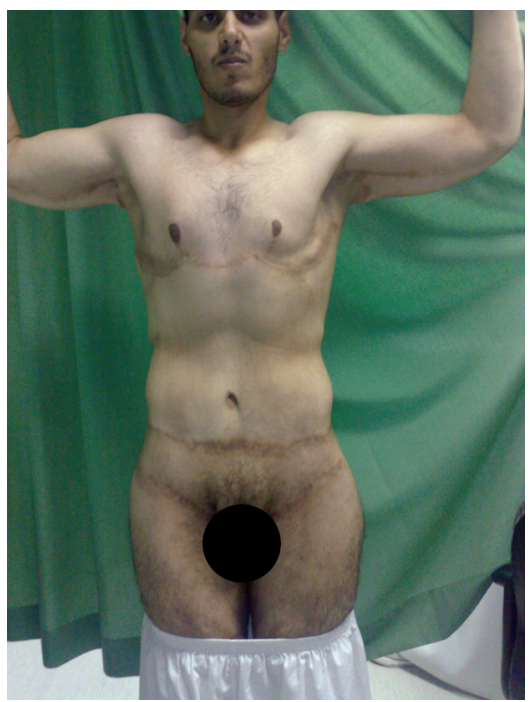

(C)

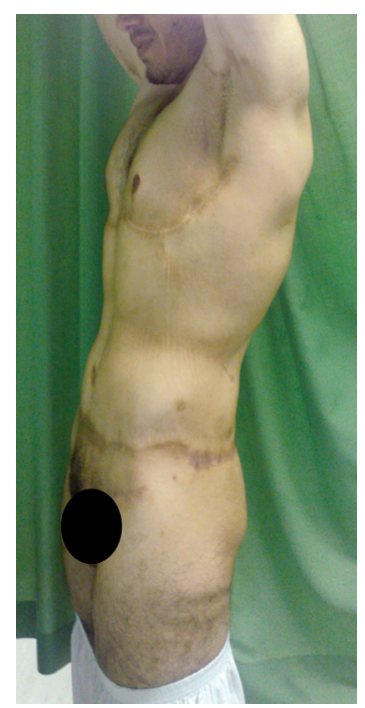

(D)

Case (5) (Group 1): Male patient 29 years old with post bariatric MWL with subsequent massive soft tissue laxity affecting his breast, abdomen, back, and anteromedial thigh. He was exposed to 3 sessions of excisional surgery. The $1^{\text {st }}$ session was belt lipectomy, the $2^{\text {nd }}$ was upper body lift, and the $3^{\text {rd }}$ was antero-medial suction assisted thigh lift plus some previous scars revisional surgery. Fig. (A) shows the patient in the $1^{\text {st }}$ sessions. Fig. (B) shows the patient in the $3^{\text {rd }}$ session with marking of suction assisted thigh lift and multiple scar revisions of previous excisional procedures. Figs. (C,D) shows the final result after the 3 sessions with multiple diverse scars that affect different areas of his body.

\section{DISCUSSION}

After FDA approved the first laser lipolysis device in 2006, rapid influx of many devices with different wavelengths $(924,968,980,1064,1319$, 1320,1344 , and $1440 \mathrm{~nm}$ ) were designated and used for lipolysis and skin tightening $[\mathbf{1 6 , 5 , 1 5 , 6 , 1 9 ]}$. Although different wavelengths were suggested however studies proved that heat, rather than a particular wavelength, led to lipolysis and tightening of the skin. On the counter side and according to some authors [4], the $1320 \mathrm{~nm}$ wavelength demonstrates greater fat absorption with less tissue penetration and scatter and therefore, may be safer for treatment around more fragile areas, such as the neck, inner thighs, and arms. In the current 
study A 1320nm wave length laser was used (CoolTouch Company, Roseville, California, USA). It proved its efficacy especially in chin, arms, back, and inner thigh.

According to Mordon's [12] mathematical analysis and other additional thermoregulatory studies [10], internal temperature between $48-50^{\circ} \mathrm{C}$ must be achieved for collagen denaturation and skin tightening and external temperatures between 38 and $41^{\circ} \mathrm{C}$ were identified as safe. Laser companies usually provide hand held or implanted sensors for measuring the external temperature [5,7], however the author never faced this problem. Just avoid long time exposure of subdermis for laser temperature.

Fast recovery, diminished postoperative pain, ecchymoses and edema are superior benefits for laser LAL over conventional ones [7,8,11]. Coagulation of blood and lymphatic vessels, aspiration of laser liquefied fat through small cannula size $(\sim 1 \mathrm{~mm})$ with subsequent less trauma may explain these benefits.

Beyond and above fat lipolysis, skin tightening effect and consequent skin reduction are perhaps the most significant advantage of laser lipolysis [1]. This last advantage is of paramount importance to improve redundant skin in massive weight loss patients. The author used LAL-ST modality in post massive weight loss patients. From analysis of data it was found that., much reduction of the incidence of excisional surgical procedures was noticed, and early post operative variable skin reduction was achieved. However, patients should remember that skin tightening continues to improve several months after laser lipolysis due to the delayed nature of neocollagenesis. Of utmost importance is to know that achievement of skin tightening equal to excisional surgery is non sense and patients should have clear explanation and should be consented for that. Better achievement may need extra session of laser skin tightening. It is essential to manage patient expectations that should be based on understanding that the skin tightening effect depend on several variables, including age, genetics, and skin condition from environmental factors, such as smoking and sun exposure. The ideal candidate for laser lipolysis is a patient who is thin, in good health, and presents with isolated pockets of removable fat [17]. In our series it was noticed that candidates with much fat were less likely to note dramatic results from single session. Evaluating the quality of the skin tone is essential in laser lipolysis. Although laser lipolysis can improve skin tone, however, it may not be able to create a completely smoothened appearance especially in exaggerated skin laxity.

The primary indication for laser lipolysis is removal of unwanted localized fat and modest skin tightening and reduction [17], also in patients with external irregularities or uneven areas after a previous liposuction or other surgical procedures, such as abdominoplasty (Fig. 3C) [5]. Surgeons are now combining procedures, such as fractional laser or radiofrequency devices, with laser lipolysis to create a synergistic effect of skin tightening [9.17]. Radiofrequency was used routinely in our cases postoperatively and improved the final results.

Beyond the standard liposuction, LAL-ST may play a unique role in certain locations: (1) Fibrous areas, such as the male breast, hips, arms, inner thigh, and back rolls (Figs. 2A,B). The smaller cannula size used for lipolysis may facilitate fat melting in fibrous locations without the additional trauma experienced with larger sized cannulas of standard liposuction, (2) It is ideal in revision surgeries where small areas of adiposity may not have been completely removed via previous lipoplasty or other body contouring procedures, (3) Lipoma removal [18], (4) Combining modalities such as combining fractional carbon dioxide laser resurfacing and laser lipolysis of the submental region to enhance neocollagenesis and skin tightening internally as well as externally [18], (5) Cellulite reduction using laser lipolysis have been documented [15]. The author used to do superficial liposuction with small cannula and low voltage laser skin tightening with the aim to improve cellulite in some patients. Much improvement was noted in a single stage procedure and some patients required extra-refinment stage.

Patients frequently present for body contouring after MWL resulting from bariatric procedures or diet and exercise. Results here showed that the number of non-bariatric patients that were exposed to LAL-ST are more in comparison to post-bariatric group. This may be related to certain reasons. AThe non-bariatric patients prefer non invasive procedures from the start, B- They are highly motivated patients than post-bariatric group, CPost-bariatric group have much skin sagging that is more candidate for excisional and lifting surgery than non-bariatric.

The disadvantages of laser lipolysis are possible localized infection, it is time consuming procedure, the cost of equipment is very high, and also reported skin burn and nerve damage due to thermolytic 
effect $[\mathbf{7 , 1 1}]$. In the author's experience, the actual complications have been rare. Future considerations will include more precise laser and light devices, improved technology, and a reduced side effect profile. For laser lipolysis specifically, treatments will be designed to optimize energy output while minimizing side effects, hastening recovery, and improving operator time.

\section{Conclusion:}

MWL population have very real aesthetic concerns that need to be addressed in an effective, definitive, and safe manner. They have variable degrees of skin redundancy and fat involution and even localized persistence of hypertrophy. The traditional ways for dealing with such aesthetic problems was excision, lifting, and even volumetric reconstruction. These procedures inevitably end up with a patient that has been transformed from a large BMI to a patient with low BMI but with the stigmata of scars. LAL-ST techniques are recent era in the field of aesthetic surgery that proved their efficacy and safety in Non-MWL body contouring. The study here proved their efficacy in MWL patients.

\section{REFERENCES}

1- Barry E. DiBernardo and Jennifer Reyes: Aesthetic Surgery Journal, September, 29 (5): 400-407, 2009.

2- De Souza Pinto E.B., Abdala P.C., Maciel C.M., et al.: Liposuction and VASER. Clin. Plast. Surg., 33 (1): $107-$ $15,2006$.

3- DiBernardo B.E. and Pozner J.N.: Principles of new invasive modalities. In: Rubin J.P., Jewell M., Richter D.F., et al., editors. Body contouring and liposuction. Philadelphia: Elsevier Saunders, p. 534-42, 2013.

4- DiBernardo B.E., Reyes J. and Chen B.: Evaluation of tissue thermal effects from 1064/1320-nm laser assisted lipolysis and its clinical implications. J. Cosmet. Laser. Ther., 11: 62-69, 2009.

5- DiBernardo B.E.: Treatment of cellulite using a 1440-nm pulsed laser with one-year follow-up. Aesthet. Surg. J., 31 (3): 328-41, 2011.

6- Elena Bravo Branas and Javier Moreno Moraga: Laser
Lipolysis Using a 924- and 975-nm Laser Diode in the Lower Extremities. Aesth. Plast. Surg., 37: 246-253, 2013.

7- Goldman A. and Gotkin R.H.: Laser-assisted liposuction. Clin. Plast. Surg., 36 (2): 241-53, vii; [discussion: 25560], 2009.

8- Heymans O., Castus P., Grandjean F.X., et al.: Liposuction: Review of the techniques, innovations and applications. Acta. Chir. Belg., 106 (6): 647-53.

9- J. Kennedy, S. Verne, R. Griffith, L. Falto-Aizpurua, K. Nouri, et al.: Non-invasive subcutaneous fat reduction: A review. J. Eur. Aca. of Derm. and Vener Sep., 29 (9): $1679-88,2015$

10- Katz B. and McBean J.: Laser-assisted lipolysis: A report on complications. J. Cosmet. Laser Ther. Dec., 10 (4): 231-3, 2008.

11- Mann M.W., Palm M.D., Sengelmann R.D., et al.: New advances in liposuction technology. Semin. Cutan. Med. Surg., 27 (1): 72-82, 2008.

12- Mordon S.R., Wassmer B., Reynaud J.P., Zemmouri J., et al.: Mathematical modeling of laser lipolysis. Biomed Eng Online. Feb., 29-7: 10, 2008.

13- Munavalli G.S. and Panchaprateep R.: Cryolipolysis for Targeted Fat Reduction and Improved Appearance of the Enlarged Male Breast. Dermatol. Surg. Sep., 41 (9): 1043$51,2015$.

14- Ortiz A.E. and Avram M.M.: Noninvasive body contouring: Cryolipolysis and ultrasound. Semin. Cutan. Med. Surg. Sep., 34 (3): 129-33, 2015.

15- Palm M.D. and Goldman M.P.: Laser lipolysis: Current practices. Semin. Cutan. Med. Surg., 28: 212-219, 2009.

16- Parlette E.C. and Kaminer M.E.: Laser-assisted liposuction: Here's the skinny. Semin. Cutan. Med. Surg., 27 (4): 25963, 2008.

17- Paul N. Afrooz, Jason N. Pozner, Barry E. DiBernardo, et al.: Noninvasive and Minimally Invasive Techniques in Body Contouring. Clin. Plastic. Surg., 41: 789-804, 2014.

18- Stebbins W.G. and Hanke C.W.: Rejuvenation of the neck with liposuction and ancillary techniques. Dermatol. Ther., 24 (1): 28-41, 2011.

19- Wolfenson M. and Norberto dos Santos Filho F.C.: Skin tightening in lipoplasty following security parameters for use of the diode laser with double wavelength $924 \mathrm{~nm}$ and $975 \mathrm{~nm}$. Plast. Reconstr. Surg. Jul., 130 (1): 235-36, 2012. 